\title{
The Role of Pharmacists in Perioperative Antibiotic Application in a Hospital of Traditional Chinese Medicine (TCM)
}

\author{
Ruifang Xie', Zhicheng Li' ${ }^{2}$, Lingqi Zhu1, Yun Zhang1, Jie Shen1, Xin Zhou ${ }^{*}$ \\ ${ }^{1}$ Department of Pharmacy, Longhua Hospital Affliated to Shanghai University of Tradtional Chinese Medcine, \\ Shanghai, China \\ ${ }^{2}$ Surgery Department, Shanghai Pudong Hospital, Shanghai, China \\ Email: ${ }^{*}$ 2479707904@qq.com
}

Received 26 December 2013; revised 7 February 2014; accepted 26 February 2014

Copyright (C) 2014 by authors and Scientific Research Publishing Inc.

This work is licensed under the Creative Commons Attribution International License (CC BY). http://creativecommons.org/licenses/by/4.0/

(c) (i) Open Access

\section{Abstract}

Objective: To determine adherence to Chinese guidelines for perioperative antibiotic use in our hospital of TCM and discuss the role of pharmacists on antibiotic management. Methods: 2844 operative cases between May 2011 and December 2011 in our hospital were investigated by pharmacists and relative data were collected, including demographic data, type of surgery, wound classification (clean and clean contaminated) and parameters of antibiotic application (antibiotic choice, route, dose, time of first dose, time of operative re-dosing and duration of prophylaxis). The rationality of antibiotic administration in these cases was respectively evaluated based on Chinese guidelines for perioperative antibiotic use. Results: After intervention of pharmacists, perioperative antibiotic application in our hospital was obviously improved from May to December of 2011: the rates of antibiotic application in operative cases and antibiotic prophylaxis in clean operation were both decreased. However, there was no significant improvement for the rate of rationality. In detail, TCM surgery was well compliant with the guidelines: the rate of antibiotic application was less than $30 \%$, regimen of antibiotic prophylaxis and therapy was reasonable; in the breast ward, antibiotic application was improved after pharmacists' intervention: the rate of antibiotic prophylaxis in clean operation significantly decreased from $100 \%$ to $30 \%$; however, in the anorectal ward, some troubles were resolved while other new problems came out; in other ward such as general surgery and orthopedics, old problems still existed. Conclusions: Protocol violations were frequent to perioperative antibiotic application in our hospital due to considerable over prescription. Intervention was effective but not enough. Further educational strategies are essential for reducing costs and antibiotic resistance rates. 


\section{Keywords}

\section{Adherence; Perioperative Antibiotic Application; Intervention}

\section{Introduction}

The contribution of antibiotic prophylaxis and therapeutics to reduce surgical site infection is well known [1]. In spite of extensive knowledge about the effectiveness of antibiotic prophylaxis and therapeutics, their administration regimens are often inappropriate. In order to reduce costs and antibiotic resistance rates and promote the rational application of antibiotics in our country, a series of documents have been officially published in recent years. All hospitals in China are urged to comply with these guidelines.

It is worth mentioning that our organization is a comprehensive hospital of traditional Chinese medicine, the herbs usage is tremendous, and some of them possess anti-microbial and anti-inflammatory functions such as Coptis Root, Polygonum Cuspidatum, Indigowoad Leaf et al. [2] [3]. So when those herbs are used in patients who have been infected, the corresponding departments may have lower rate of antibiotics use.

Based on the above background, the appropriateness of antibiotic application in our hospital, especially perioperative antibiotics, was selected as a focus. The operative cases in our hospital were investigated and antibiotic application was evaluated according to published guidelines and related literatures. Meanwhile, the effects of herbs to antibiotics usage were discussed.

\section{Data and Methods}

\subsection{Data Collection}

All operative patients' records in our hospital between May 2011 and December 2011 were observed. For our research purposes, a case record form was developed which included information on patient characteristics, surgical procedures, laboratory results and antibiotic treatments for each subject. Wound classification was obtained from the antibiotic ordering form which was sent to the hospital pharmacy for each patient prior to surgery. When the wound classification was not reported, the wound was classified from the operation report according to the China standards for that type of surgical procedure. The following aspects of antibiotic treatments were examined: the antibiotic agent, the route of administration, the timing of operative re-dosing and the duration of antibiotic application.

\subsection{Assessment Criteria}

The appropriateness of antibiotic application was evaluated in accordance with the following documents: 1) Notification about issues related to management of antibiotics clinical application composed by General Office of the Ministry of health in 2009 (NO. 38); 2) Guidelines for antimicrobial application drafted by the Chinese medical association, the Chinese hospital association and the Chinese pharmaceutical association; 3) information from medication package inserts.

The main points of antibiotics application guidelines (NO. 38) are as follows: 1) I class incision surgery generally doesn't use antimicrobial drugs for prophylaxis. Otherwise, corresponding indication, drug choice, drug initiation and duration time must be controlled strictly. Dosing method accords to the relevant regulations: the first time delivery must be within 0.5 to 2 hours before surgery or at the beginning of anesthesia. When operation time is more than $3 \mathrm{~h}$ or blood loss is greater than $1500 \mathrm{~mL}$, the second agent drug can be given, and total prophylaxis time is generally not more than $24 \mathrm{~h}$, but individual cases can be extended to $48 \mathrm{~h}$. 2) Strictly control fluoroquinolone drugs in clinical application: strictly gasp clinical application indications and control number of species. Empirical treatment of fluoroquinolone drugs can be used in intestinal infection, community acquired respiratory tract infections, and community acquired urinary tract infection. 3) Strictly perform the classification management of antimicrobial drugs, include unrestricted use, restricted use and special use. 4) Establish the early warning system for the clinical application of antimicrobial drugs, and take appropriate intervention measures. For the main target bacteria, when resistance rate of antimicrobial drugs is more than $30 \%$, the early warning information should be timely passed to agency staff; more than $40 \%$, empirical usage should be cau- 
tious; more than $50 \%$, drugs should be selected according drug sensitive test results; more than $75 \%$, these drugs use should be suspended, and the decision whether to restore its clinical application in accordance with the tracking monitoring results of bacterial drug resistance.

\subsection{Protocol}

The required data were provided by the statistics department. The pharmacists assessed the appropriateness of antibiotic application according to the above criteria, summarized and submitted reports to the department of medical management each month. In our study three parameters from each month such as antibiotic using rate, antibiotic use rate in clean operations and rate of unreasonable antibiotic use were compared. Controversial issues were arbitrated by the group of bacterial drug experts which consisted of experienced doctors and pharmacists. The department of medical management checked the reports, notified the clinics and interfered in unreasonable administrations together with pharmacists when necessary (Figure 1).

\subsection{Analyses}

Data were entered into EXCEL and analyzed by SPSS16.0.

\section{Results}

Between May 2011 and December 2011, 2844 patients were enrolled during our survey period. Ophthalmology operations were excluded because a consensus about the antibiotic prophylaxis of ophthalmology had yet to be agreed upon. Of the 2844 patients, 1217 (42.87\%) underwent a clean surgical procedure while all others received a clean-contaminated or contaminated operation. The majority of procedures were carried out by the anorectal ward (33.30\%), followed by the breast ward (20.39\%), orthopedics (14.59\%) and the general surgery ward (11.99\%) (Figure 2). This showed that the four wards were important operative departments. As a result, they should be paid more attention.

Perioperative antibiotic use in the whole hospital was improved during several months last year. The rates of antibiotic application and antibiotic prophylaxis for clean operations both obviously decreased from May to December while the rate of unreasonable application fluctuated (Table 1, Figure 3). The details of various wards are shown below:

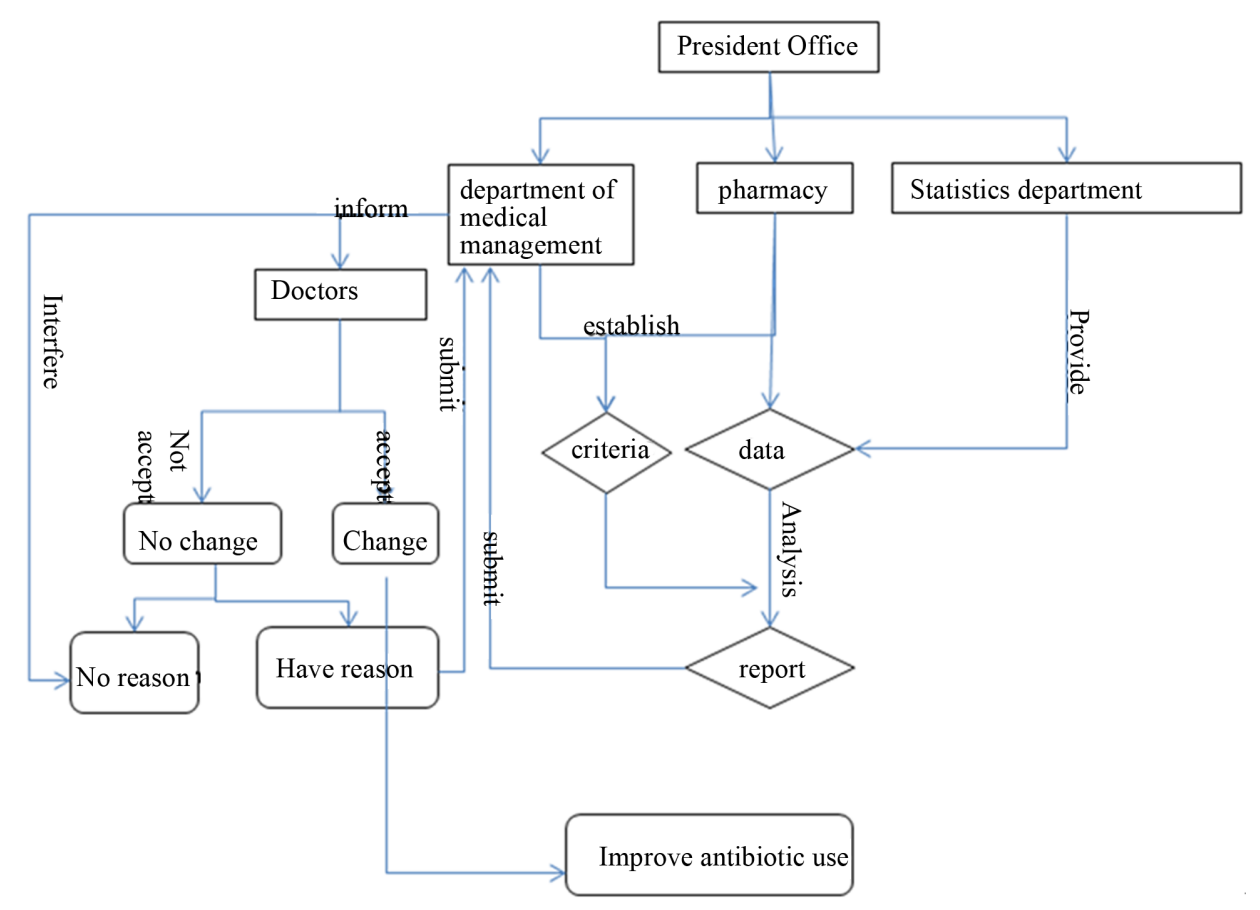

Figure 1. Management process of perioperative antibiotic application in our hospital. 
Table 1. Antibiotic application between May and December in 2011.

\begin{tabular}{cccc}
\hline Months & $\begin{array}{c}\text { Rate of antibiotic } \\
\text { application }\end{array}$ & $\begin{array}{c}\text { Rate of antibiotic application } \\
\text { for clean opaeration }\end{array}$ & $\begin{array}{c}\text { Unreasonable rate of } \\
\text { antibiotic application }\end{array}$ \\
\hline May & $85.48 \%$ & $84.44 \%$ & $38.63 \%$ \\
June & $86.98 \%$ & $83.11 \%$ & $55.33 \%$ \\
July & $83.00 \%$ & $83.23 \%$ & $35.13 \%$ \\
August & $81.69 \%$ & $88.28 \%$ & $40.71 \%$ \\
September & $80.43 \%$ & $73.25 \%$ & $45.65 \%$ \\
October & $77.12 \%$ & $67.59 \%$ & $50.47 \%$ \\
November & $73.61 \%$ & $56.44 \%$ & $46.17 \%$ \\
December & $69.27 \%$ & $56.21 \%$ & $53.15 \%$ \\
\hline
\end{tabular}

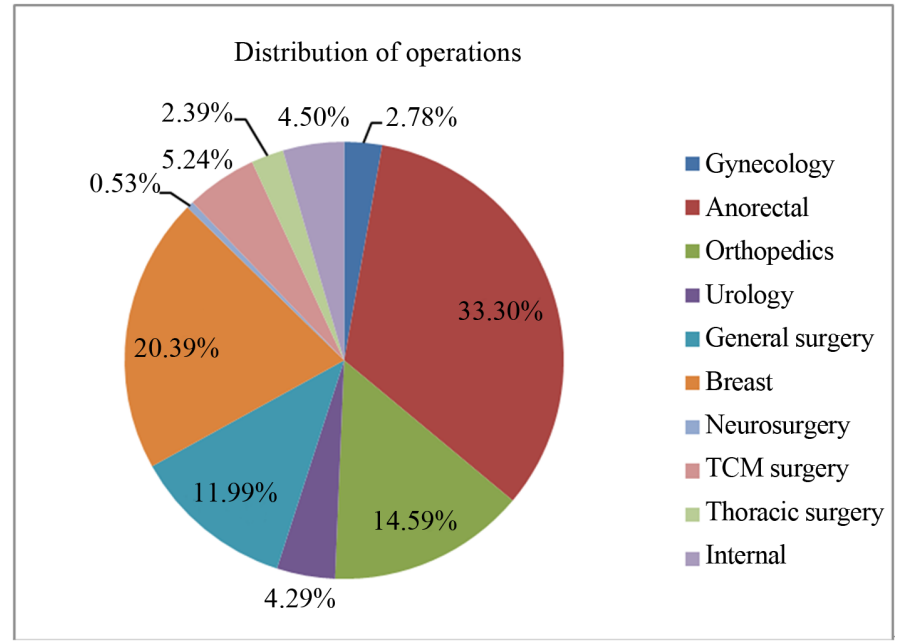

Figure 2. Distribution of operations in our hospital between May and December in 2011.

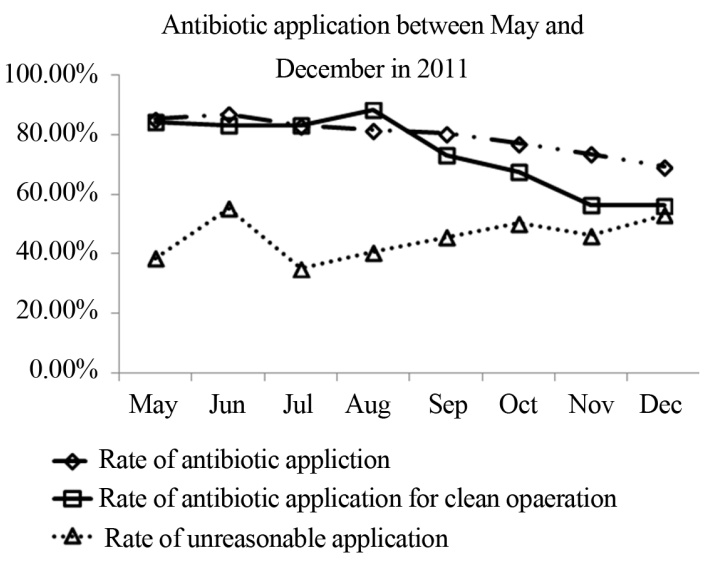

Figure 3. Antibiotic application between May and December in 2011.

In the breast ward, intervention for perioperative antibiotic use was very effective (Table 2, Figure 4): Antibiotics were administered to almost all patients undergoing breast operations before August in spite of wound 
Table 2. Antibiotic application of surgical departments between May and December in 2011.

\begin{tabular}{|c|c|c|c|c|c|c|}
\hline \multirow{2}{*}{ Rate } & \multirow{2}{*}{ Months } & \multicolumn{5}{|c|}{ Surgical wards } \\
\hline & & Breast & General surgery & Orthopedics & Anorectal & TCM surgery \\
\hline \multirow{9}{*}{$\begin{array}{c}\text { Rate of } \\
\text { Antibiotic } \\
\text { application }\end{array}$} & May & $98.51 \%$ & $82.69 \%$ & $78.72 \%$ & $96.24 \%$ & $31.25 \%$ \\
\hline & Jun & $100.00 \%$ & $84.78 \%$ & $71.93 \%$ & $96.75 \%$ & $25.00 \%$ \\
\hline & Jul & $100.00 \%$ & $84.21 \%$ & $85.71 \%$ & $91.96 \%$ & $13.33 \%$ \\
\hline & Aug & $100.00 \%$ & $80.00 \%$ & $97.96 \%$ & $84.56 \%$ & $20.69 \%$ \\
\hline & Sep & $77.92 \%$ & $78.13 \%$ & $78.85 \%$ & $94.79 \%$ & $26.67 \%$ \\
\hline & Oct & $58.62 \%$ & $86.54 \%$ & $82.98 \%$ & $99.03 \%$ & $21.05 \%$ \\
\hline & Nov & $43.06 \%$ & $68.00 \%$ & $90.74 \%$ & $98.47 \%$ & $25.00 \%$ \\
\hline & Dec & $36.47 \%$ & $78.05 \%$ & $88.33 \%$ & $99.12 \%$ & $22.22 \%$ \\
\hline & May & $100.00 \%$ & $72.22 \%$ & $78.72 \%$ & - & $100.00 \%$ \\
\hline \multirow{7}{*}{$\begin{array}{l}\text { Rate of } \\
\text { antibiotic } \\
\text { application } \\
\text { for clean } \\
\text { opaeration }\end{array}$} & Jun & $100.00 \%$ & $76.92 \%$ & $70.37 \%$ & - & $40.00 \%$ \\
\hline & Jul & $100.00 \%$ & $70.59 \%$ & $85.71 \%$ & - & $0.00 \%$ \\
\hline & Aug & $100.00 \%$ & $53.85 \%$ & $97.96 \%$ & - & $0.00 \%$ \\
\hline & Sep & $76.67 \%$ & $64.71 \%$ & $78.85 \%$ & - & $20.00 \%$ \\
\hline & Oct & $55.77 \%$ & $68.18 \%$ & $83.67 \%$ & - & $25.00 \%$ \\
\hline & Nov & $32.79 \%$ & $48.00 \%$ & $90.57 \%$ & - & $0.00 \%$ \\
\hline & Dec & $31.08 \%$ & $62.50 \%$ & $88.14 \%$ & - & $33.33 \%$ \\
\hline \multirow{8}{*}{$\begin{array}{c}\text { Rate of } \\
\text { unreasonable } \\
\text { application }\end{array}$} & May & $71.64 \%$ & $26.92 \%$ & $8.51 \%$ & $42.11 \%$ & $6.25 \%$ \\
\hline & Jun & $90.00 \%$ & $43.48 \%$ & $17.54 \%$ & $66.67 \%$ & $8.33 \%$ \\
\hline & Jul & $75.64 \%$ & $21.05 \%$ & $12.24 \%$ & $32.14 \%$ & $0.00 \%$ \\
\hline & Aug & $83.56 \%$ & $46.67 \%$ & $14.29 \%$ & $32.35 \%$ & $0.00 \%$ \\
\hline & Sep & $55.84 \%$ & $21.88 \%$ & $7.69 \%$ & $69.79 \%$ & $13.33 \%$ \\
\hline & Oct & $36.21 \%$ & $25.00 \%$ & $23.40 \%$ & $99.03 \%$ & $10.53 \%$ \\
\hline & Nov & $25.00 \%$ & $18.00 \%$ & $5.56 \%$ & $98.47 \%$ & $12.50 \%$ \\
\hline & Dec & $27.06 \%$ & $53.66 \%$ & $30.00 \%$ & $99.12 \%$ & $0.00 \%$ \\
\hline
\end{tabular}

Antibiotic application in breast ward

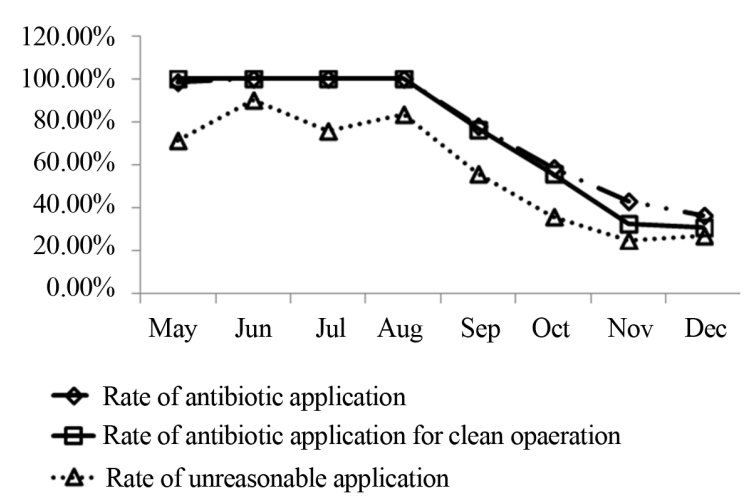

Figure 4. Antibiotic application in breast ward. 
classification. However, after pharmacists' intervention, the rate of antibiotic prophylaxis and therapeutics gradually decreased until about 30\% in December. Meanwhile, the rate of inappropriate application was significantly reduced from $71.64 \%$ to $27.06 \%$ between May and December.

In the TCM surgery, some of the primary diseases treated were diabetic gangrene, erysipelas, ulceration, and snake bite, so a large part of operations involved incision, drainage and debridement of skin and soft tissue, which were catalogued into contaminated procedures. However, to our pleasure, antibiotic protocols in this ward were always satisfied while the risk of surgical site infection did not obviously increase. The rate of antibiotic application was low (<30\%); although the antibiotic use rate in clean operations was $100 \%$ in May, the reason was that only one clean procedures were executed in that month and antibiotics were seldom administrated for these operations; furthermore, most of antibiotics were properly performed (Table 2, Figure 5). As we know, herbs are commonly given to patients orally or externally after procedures in this ward and characteristics of TCM might be associated with the low rates of antibiotic use and adherence to relevant guidelines in this department. It may indicate that chemical antibiotics might not be the sole agents able to prevent surgical site infection and some herbs might also possess antimicrobial activities [4] [5].

In general surgery and orthopedics, the parameters of antibiotic use fluctuated (Table 2, Figures 6, 7) and some problems existed:

1) No indications. For example, thyroidectomy is considered a clean operation and is not suitable for antibiotic prophylaxis [6]. Despite this, in some cases antibiotics were used.

2) Unsuitable selection of drug. The first or second generation cephalosporins were normally chosen for clean or clean-contaminated procedures [7]. If a patient could not safely receive cephalosporin because of an allergy, clindamycin and aztreonam were used separately for gram positive and gram negative bacterium. However, in some operations of orthopedics, such as implant fixation of fractures, fluoroquinolones were often selected for

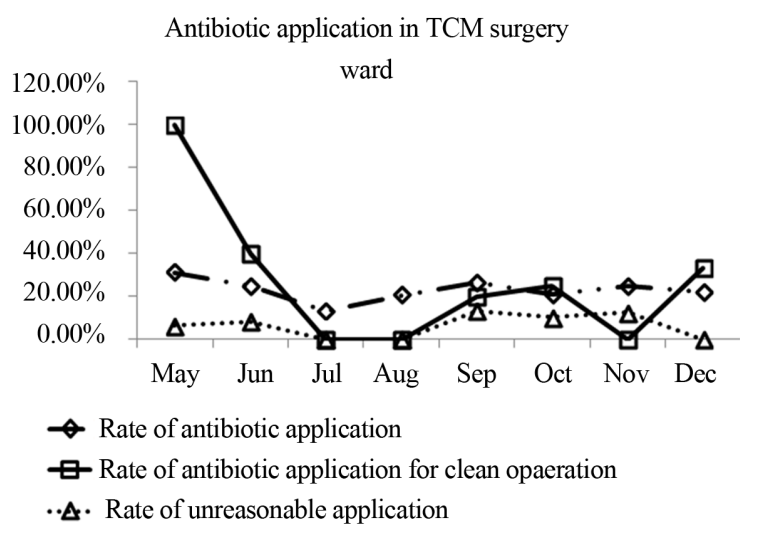

Figure 5. Antibiotic application in TCM surgery ward.

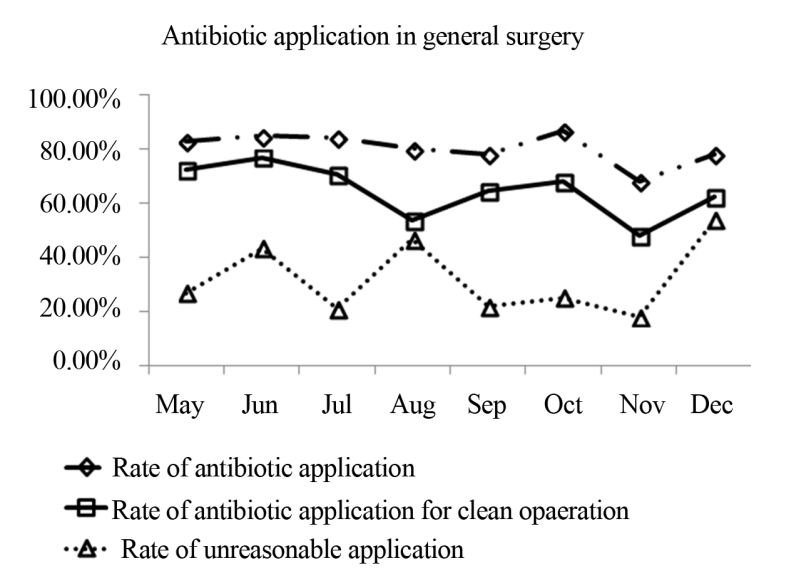

Figure 6. Antibiotic application in general surgery. 


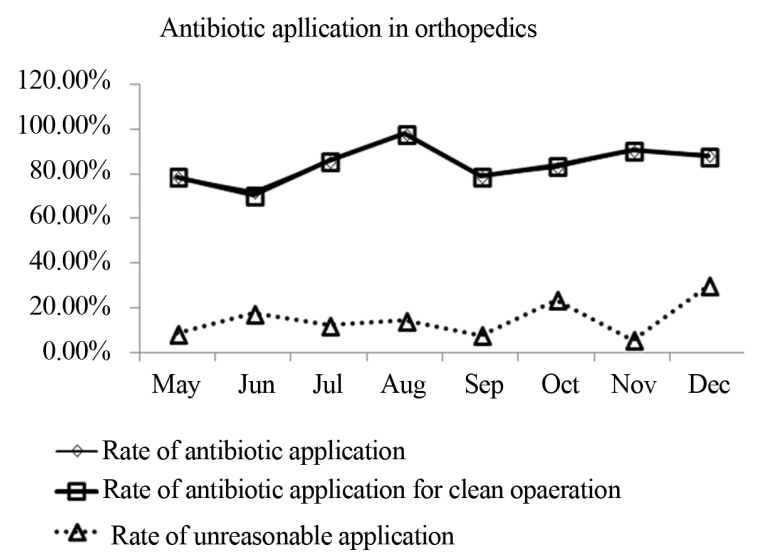

Figure 7. Antibiotic application in orthopedics.

prophylaxis. This unreasonable selection increased emergence of fluoroquinolone resistance.

3) Incorrect timing of administration. Antibiotic prophylaxis was normally used to ensure antibiotic presence in adequate concentration in the targeted tissue throughout the operative period [8]. The guidelines for antibiotic management in our country recommend antibiotics should be given at the beginning of induction or half to two hours before operation. However, in some cases the first dose of antimicrobial agent was administrated to patients after operation.

4) Prolongation of antimicrobial application. According to the guidelines for antibiotic management and literature duration of appropriate antibiotic prophylaxis should be less than $24 \mathrm{~h}$ and may be prolonged to $48 \mathrm{~h}$ under some special conditions. There was no evidence that prolongation in postoperative period decreased the incidence of surgical site infection [9] [10]. In cases of prolonged surgery ( $>3 \mathrm{~h})$ and excessive bleeding (>1.5 L), intra-operative re-administration was indicated. However, in some cases, duration of some procedures such as hernia repair and implant fixation of fractures exceeded $48 \mathrm{~h}$ and even longer without explicit indications.

5) Overdose of antibiotic agents. According to the recommendations in the guidelines, preventative dosages of cefuroxime and metronidazole were respectively $1.5 \mathrm{~g}$ and $0.5 \mathrm{~g}$, but in some records actual amounts were overdosed with cefuroxime ( $2.25 \mathrm{~g}$ ) and metronidazole ( $2 \mathrm{~g})$, leading to additional cost and adverse reactions.

6) Inappropriate combination of drugs. For instance, cefmetazole and ornidazole were combined together in several surgical patients. Both of drugs are active against anaerobic bacteria. It was unnecessary for patients to be exposed to both medcines at the same time only for prophylaxis.

7) No clear reason for drug replacement. In individual cases, cefuroxime and metronidazole before operation were replaced by cefotiam and ornidazole after the procedure. Both cefuroxime and cefotiam are classified as second generation cephalosporins, furthermore metronidazole and ornidazole are both nitromidazoles. These drugs' antimicrobial spectrums are similar, but there was no indication for the replacement noted in the patients' medical records, indicating replacement of drugs was casual.

8) Inaccurate frequency of administration. For the sake of convenience, some doctors were not compliant with the pharmacokinetics of the antibiotic used. For example, cefotiam doses should be given divided two to four times per day according to the information provided by the manufacturer. In some cases cefotiam was given once daily, which may have led to decreased efficacy.

In the anorectal ward, a large part of operations were fistulectomy and haemorrhoidectomy, which classified as contaminated operations or dirty wounds. During the period of observation, some problems were resolved while new troubles came out, which contributed to the fluctuation of parameters (Table 2, Figure 8).

1) In May, June and July, pazufloxacin was widely used. Pazufloxacin belongs to the fluoroquinolone drug class, and is not suitable for operative prophylaxis. Following intervention by pharmacists, improvement was not obvious. Due to this, a report was submitted to the department of medical management and pazufloxacin was removed from our hospital formulary. Following this intervention, the situation improved.

2) In the next months, ornidazole was generally chosen to prevent anaerobic bacterial infections. With regard to the cost, the medical insurance rules of Shanghai noted that ornidazole should only be given to patients who cannot tolerate the adverse reactions of metronidazole. The investigators checked patients' histories, but found 


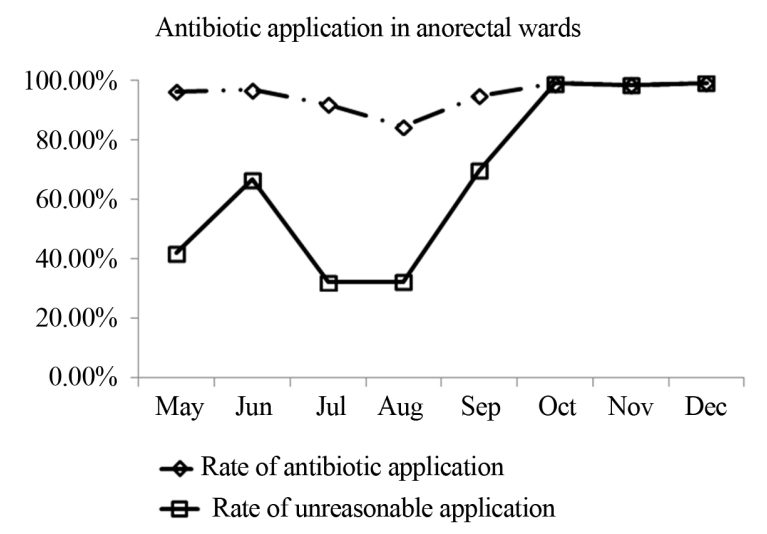

Figure 8. Antibiotic application in anorectal ward.

no records of indicating that patients were intolerant adverse reactions of metronidazole. Due to this, the investigators negotiated with the clinical doctors. The physicians argued that because postoperative patients often felt gastrointestinal discomfort due to anaesthesia and analgesia pumps, it was necessary to select ornidazole instead of metronidazole. An agreement seemed to be reached.

In other ward, similar problems were also exhibited:

1) In the urological surgical ward, aztreonam was commonly selected for antibiotic prophylaxis despite patients' allergic history. Based on recommendations for antibiotic management, aztreonam should only be used for patients who are allergic to penicillin. Selection of drugs should be made according to patients' allergy histories.

2) In the thoracic surgery ward, cefoxitin, a cephamycin, was administrated once daily for prophylaxis. To our knowledge, the choice of this drug and frequency of administration were incorrect.

3) In the gynecology ward, problems such as inappropriate combination of drugs and incorrect timing of administration were also sometimes present.

\section{Discussion}

Our hospital is affiliated to shanghai university of Traditional Chinese Medicine and the specialty is Traditional Chinese Medicine, which might contribute to the types of operations normally uncomplicated and low risk. As showed above, a large part of operations was breast surgery, thyrodectomy, inguinal hernia repair, excision of hemorrhoids and so on. This suggested that there was plenty more for pharmacists to improve perioperative antibiotic use.

In 2011 pharmacists made great efforts backed by president office and department of medical management. After several months, obvious improvements were shown in some wards, especially breast ward. In other wards improvements were not significant. This demonstrated that our jobs were effective but required further improvements. Some problems were presented as follows:

1) Some wounds were not clearly classified. As a result, it was difficult to speculate antibiotics use as therapy or prophylaxis. In some anorectal operations such as excision of hemorrhoids and perianal abscess, it was often confused whether antibiotics were used as prophylaxis or therapy. Doctors and pharmacists should communicate more frequently and reach a consensus.

2) Unclear and wrong records brought out some problems for pharmacists to judge the rationality of antibiotic use. Administration time points of antibiotics were often omitted, which was not convenient for us to evaluate the propriety of time; progress of diseases was sometimes ambiguous, which puzzled us to find the reasons of drug replacements; classification of wounds in operation records was inconsistence with discharge summary ect. These indicated that the department of medical management should pay attention to the quality of case history.

3) Similar problems seemed to come up again and again in some wards. This indicated that the interventions were not enough. These difficulties should be resolved.

Of course this paper was limited. It was retrospective study and all interventions were performed afterwards. In the future additional measures should be carried out: antibiotic management software should be implanted in 
computer system (the Hospital Information system); contacts between doctors and pharmacists should be closer for consensus on lots of issues; authorities should take more effective methods to achieve optimal adherence, ect. Through these efforts by clinics and pharmacists, continuous improvement in perioperative antibiotic use of our hospital is possible in the future.

\section{Acknowledgements}

This work was financially supported by Longhua Medicial Project (LYTD-14) and Shanghai Pharmaceutical Association fund (No. 2011-YY-03-12)

\section{References}

[1] Gagliardi, A.R., Fenech, D., Eskicioglu, C., et al. (2009) Factors Influencing Antibiotic Prophylaxis for Surgical Site Infection Prevention in General Surgery: A Review of the Literature. Canadian Journal of Surgery, 52, 481-489.

[2] Stermitz, F.R., Lorenz, P., Tawara, J.N., et a1. (2000) Synergy in a Medicinal Plant: Antimicrobial Action of Berberine Potentiated by 5'-Methoxyhydnocarpin, a Multidrug Pump Inhibitor. PNAS, 97, 1433-1437.

[3] Cai, Y., Xing, Y. and Xie, W.G. (2011) Rearch on Progress of Antibacterial Activity of Chinese Medicine. China Medical Herald, 8, 9-10.

[4] Kwan, H.A., Kwon, Y.-J., Kwon, D.-Y., et al. (2008) Evaluation of Antibacterial Effects of a Combination of Coptidis Rhizoma, Mume Fructus, and Schizandrae Fructus against Salmonella. International Journal of Food Microbiology, 127, 180.

[5] Jayampath Seneviratne, C., Ricky, W.K.W. and Lakshman, P.S. (2008) Potent Anti-Microbial Activity of Traditional Chinese Medicine Herbs against Candida Species. Mycoses, 51, 30-34.

[6] Tourmousoglou, C.E., Yiannakopoulou, E.C., Kalapothaki, V., et al. (2008) Adherence to Guidelines for Antibiotic Prophylaxis in General Surgery: A Critical Appraisal. Journal of Antimicrobial Chemotherapy, 61, 214-218.

[7] Nicaragua, J., Disseldorp, V., Slingenberg, E.J.M.H., et al. (2006) Application of Guidelines on Preoperative Antibiotic Prophylaxis in León. The Netherlands journal of medicine, 64, 411-416.

[8] Enzler, M.J., Berbari, E. and Osmon, D.R. (2011) Antimicrobial Prophylaxis in Adults. Mayo Clinic Proceedings, 86, 686-701.

[9] Velmahos, G.C., Toutouzas, K.G., Sarkisyan, G., et al. (2002) Severe Trauma Is Not an Excuse for Prolonged Antibiotic Prophylaxis. Archives of surgery, 137, 537-542.

[10] Dechiara, S., Chiumello, D., Nicolini, R., et al. (2010) Prolongation of Antibiotic Prophylaxis after Clean and Clean-Contaminated Surgery and Surgical Site Infection. Minerva Anestesiologica, 76, 413-419. 